\title{
Begleitung traumatisierter Frauen während der Geburt
}

\author{
Martina Kruse, Hebamme, Familienhebamme, Systemische Beraterin und Traumazentrierte Fachberaterin, Köln
}

\begin{abstract}
Jede Hebamme begleitet im Laufe ihrer Berufstätigkeit wissentlich oder unwissentlich auch Frauen durch Schwangerschaft und Geburt, die aus unterschiedlichen Gründen an den Folgen eines Traumas leiden. Unsere Autorin ging in ihrer Masterarbeit der Frage nach, was Hebammen benötigen, um diese nicht einfache Aufgabe zum Wohle der ihnen anvertrauten Frauen zu erfüllen. In diesem Beitrag fasst sie wichtige Voraussetzungen für eine traumasensible Arbeits- und Sichtweise in Gesundheitsberufen zusammen.
\end{abstract}

Oft ist nicht auf den ersten Blick ersichtlich, dass eine Frau aufgrund eines früheren Erlebens traumatisiert ist. Umso wichtiger ist es, auf mögliche Anzeichen dafür zu achten: Lässt die Frau die Geburt klaglos über sich ergehen? Wirkt sie dabei wie abgeschaltet, abwesend? Oder gerät eine Frau, obwohl bei der Geburt zunächst alles ganz normal verläuft, irgendwann völlig außer sich und ist fürs geburtshilfliche Personal nicht mehr erreichbar? In diesem Fall endet die Geburt vielleicht operativ und es stellt sich die Frage: warum eigentlich? Weil ein früheres Trauma solch weitreichende Folgen haben kann, sollten Hebammen die Möglichkeiten kennen, diese Frauen gut und sicher durch die Geburt zu leiten.

\section{Definition Trauma}

\author{
Merke \\ Ein Trauma ist „ein vitales Diskrepanzerlebnis zwischen \\ bedrohlichen Situationsfaktoren und den individuellen \\ Bewältigungsmöglichkeiten, das mit Gefühlen von Hilf- \\ losigkeit und schutzloser Preisgabe einhergeht und so \\ eine dauerhafte Erschütterung von Selbst- und Weltver- \\ ständnis bewirkt" [4].
}

Bei einem Trauma handelt es sich um ein einschneidendes, die Existenz eines Menschen bedrohendes Ereignis. Ob etwas als Trauma erinnert wird oder nicht, ist ein subjektives Erleben. Entscheidend ist, ob und welche Strategien der Person zur Bewältigung zur Verfügung stehen und dass diese genutzt werden können. Wer sich in einem solchen Moment als hilflos und ohnmächtig empfindet, sieht keine Handlungsmöglichkeiten. Dann ist die Wahrscheinlichkeit sehr hoch, dass das Geschehen als Trauma erinnert wird. Ein weiteres Kriterium entsprechend dieser Definition ist das, was infolge des Ereignisses passiert: Die veränderte Sicht der Person auf sich selbst und auf die Welt. Viele Betroffene beschreiben das sehr passend mit dem Satz: „Danach war nichts mehr so wie vorher!“

\section{Beispiel}

Wie eine Person ein Ereignis erlebt, ist entscheidend für dessen Verarbeitung und mögliche Folgen. So kann ein und dasselbe Ereignis - zum Beispiel ein Überfall - für eine Frau weitreichende Folgen haben, für eine andere aber nach einer kurzen Zeit der Belastung ohne Auswirkungen bleiben.

Die erste Frau in dem Beispiel hatte im Moment des Übergriffs vielleicht die Möglichkeit, für sich zu sorgen und zu handeln, indem sie sich in Sicherheit bringen oder fliehen konnte. Sie fühlt sich danach einige Zeit belastet und gestresst, hat aber nach einer Weile das Gefühl, dass alles wieder seinen Gang geht. Ihr Leben hat sich nach dem Überfall nicht gravierend geändert. Für sie ist die Welt weiter ein sicherer Ort.

Die andere Frau in dem Beispiel hatte dagegen das Gefühl, handlungsunfähig zu sein und keinen Einfluss auf die Situation zu haben. Sie erlebte sich als ohnmächtig. Sie geht nach dem Vorfall nach Hause und findet nicht mehr zurück in ihr unbeschwertes Leben. Sie zieht sich zurück, ist schreckhaft, hat vielleicht Alpträume. Ihre Welt hat sich langfristig verändert.

\section{Prävalenz}

Nicht jedes traumatische Erleben muss Folgen haben. Schätzungen gehen davon aus, dass circa $50 \%$ aller Menschen in ihrem Leben eine traumatische Erfahrung machen. Ungefähr 30\% leben ihr Leben nach einer kurzen Zeit der Anpassung unbeeinträchtigt weiter. Weitere 30\% scheinen stabil - diese Stabilität kann allerdings zusammenbrechen, sobald eine weitere Belastung auftritt. Das kann beispielsweise eine Schwangerschaft sein. Das letzte Drittel benötigt zur Integration des Ereignisses von vornherein Unterstützung. Diese Menschen entwickeln eine Posttraumatische Belastungsstörung (PTBS) [8]. Für Deutschland wird eine Lebenszeitprävalenz von 1,5-2\% 
angegeben, wobei Frauen häufiger betroffen sind als Männer. Die Wahrscheinlichkeit, mit der man nach einem bestimmten Ereignis Symptome einer PTBS zeigt, wird unterschiedlich hoch angegeben.

Eine 50-prozentige Wahrscheinlichkeit, an einer PTBS zu erkranken, besteht bei Menschen, die Krieg und Folter oder eine Vergewaltigung erlebt haben. Andere Gewalttaten oder schwere Erkrankungen haben ein etwas geringeres Risiko [5]. Zu den typischen Merkmalen einer PTBS werden Intrusionen gezählt. Darunter versteht man Erinnerungsblitze in Form von Flashbacks, Bildern oder Alpträumen. Dies sind nicht wirklich Erinnerungen im Sinne von, „das ist vor langer Zeit passiert“, sondern sie stellen das Wiedererleben der traumatischen Situation dar. Weiter besteht eine Übererregbarkeit, eine dauerhafte Anspannung, die sich zum Beispiel durch Schlafstörungen, Konzentrationsschwierigkeiten oder leichte Reizbarkeit äußert (Hyperarousel). Die Betroffene zieht sich in sich zurück, versucht bestimmte (mit dem Trauma verbundene) Orte, Personen, Situationen oder Empfindungen zu meiden. Sie fühlt sich von sich und von anderen entfremdet und meidet vielleicht soziale Kontakte (Konstriktion) [5].

\section{Ursachen}

Die Ursachen für Traumata sind vielfältig:

- Sexualisierte oder körperliche Gewalt: Jede dritte Frau hat seit ihrem 15. Lebensjahr körperliche Gewalt erfahren, jede fünfte Frau körperliche und/oder sexualisierte Gewalt innerhalb einer Partnerschaft. $5 \%$ der Frauen sind seit dem 15. Lebensjahr vergewaltigt worden und jede dritte Frau hat in der Kindheit körperliche und/oder sexualisierte Gewalt erfahren [5].

- Schwere Erkrankungen, Unfälle, medizinische Eingriffe

- Traumatische Geburtserlebnisse: Sowohl die Geburt als auch notwendige Interventionen oder die Betreuung der Hebammen und Geburtshelfenden können hier verursachend sein. Schätzungen gehen davon aus, dass 1,5 - 6\% der Mütter postpartal Symptome einer PTBS entwickeln [2].

- Kindesvernachlässigung und -misshandlung

- Kriege, Vertreibung, Folter

- Weibliche genitale Verstümmelung (FGM)

- Verlust einer Bezugsperson, insbesondere eines Elternteils im Kindesalter

- Miterleben von Gewalt als Zeugin (Kind, Partnerin, Angehörige von helfenden/pflegenden Berufen)

- Naturkatastrophen

- Konfrontation mit Traumafolgen als Helferin (stellvertretendes Trauma)

In der 1993 von der Generalversammlung der Vereinten Nationen verabschiedeten „Erklärung über die Beseitigung von Gewalt gegen Frauen“ wird insbesondere geschlechtsspezifische Gewalt definiert. Demnach ist Gewalt gegen Frauen ,jede gegen Frauen aufgrund ihrer Geschlechtszugehörigkeit gerichtete Gewalthandlung, durch die Frauen körperlicher, sexueller oder psychologischer Schaden oder Leid zugefügt wird oder werden kann, einschließlich der Androhung derartiger Handlungen, der Nötigung oder der willkürlichen Freiheitsberaubung, sowohl im öffentlichen als auch im privaten Bereich“[12].

\section{Physiologie}

Was aber passiert während eines traumatisch erlebten Ereignisses im Körper? Im Falle einer bedrohlichen Situation startet das Gehirn ein Notfallprogramm. Im Körper werden Hormone (Adrenalin, Noradrenalin und Cortisol) freigesetzt, die den Menschen auf Kampf oder Flucht vorbereiten. Der Herzschlag beschleunigt sich, Energie wird freigesetzt. Sind Kampf und Flucht unmöglich, erstarrt der Betroffene. Das Gedächtnis funktioniert in diesem Fall verändert. Die Verbindung zwischen Hippocampus und Großhirnrinde ist unterbrochen, sodass Erinnerungen nicht mehr biographisch korrekt abgespeichert werden. Stattdessen werden Erinnerungsfetzen, sogenannte Fragmente, im Gedächtnis abgelegt. Erinnert werden dann z.B. nur Gerüche, visuelle Eindrücke, Worte oder Körperempfindungen, aber nicht mehr das gesamte Bild - es fehlt die zeitliche Zuordnung. Es ist nicht möglich, zusammenhängend vom Ereignis zu berichten. Manche Erinnerungen werden abgespalten - dissoziiert.

Das Traumagedächtnis und die Körpererinnerung sind allerdings leicht triggerbar: Hört jemand lange Zeit später das gleiche Wort oder nimmt das gleiche Gefühl wahr, löst das im Gehirn sofort die gleichen Reaktionen aus wie in der früheren traumatischen Situation. Das Gehirn unterscheidet nicht zwischen gefährlich oder ungefährlich, zwischen damals und heute. Der Trigger (Auslöser) bewirkt, dass der Betroffene so reagiert, als wäre er wieder in der lebensbedrohlichen Situation [1]. Er erlebt einen Flashback.

Schwangerschaft und Geburt nach früheren traumatischen Erfahrungen können für Frauen zu einer besonderen Herausforderung werden. Dies muss aber nicht zwangsläufig der Fall sein. Ein großer Teil der Frauen erlebt diese Zeit ähnlich wie jede nicht betroffene Frau - als aufregend, anstrengend und fordernd. Diese Frauen spüren ihre Kraft und ihre Fähigkeit, Leben hervorzubringen.

\footnotetext{
Merke

Um Frauen angemessen während der Geburt begleiten zu können, ist es notwendig, die körperlichen Vorgänge des Traumaerlebens zu kennen. Mit diesem Wissen lassen sich die beobachtbaren Reaktionen entsprechend einordnen, sodass angemessen darauf reagiert werden kann.
} 


\section{Anzeichen erkennen}

Wie lässt sich erkennen, ob eine Gebärende an Traumafolgen leidet? Eine Möglichkeit besteht darin, im Rahmen der Anamnese nach belastenden Ereignissen zu fragen.

Gewalt als häufige Ursache von Traumata hat deutliche Auswirkungen auf die Frauengesundheit, auf das Erleben von Schwangerschaft, Geburt und Mutterschaft $[6,9]$. Dennoch werden Frauen nicht regelmäßig danach gefragt. Dabei zeigen Untersuchungen, dass Frauen durchaus darauf angesprochen werden möchten: NichtBetroffene fühlen sich dadurch nicht beeinträchtigt, Betroffene hätten andernfalls vielleicht nicht den Mut, das Erlebte von sich aus zu thematisieren [7]. Es ist also nicht die Frage $o b$, sondern wie und wann nach Gewalterfahrungen gefragt wird.

Vielleicht ergibt sich nicht im ersten Gespräch eine Möglichkeit dazu, vielleicht bietet sich erst ein späterer Termin dafür an. In einem vertraulichen Setting, bei einem Gespräch auf Augenhöhe - und das ist wörtlich zu verstehen - könnte die Hebamme zum Beispiel folgendermaßen ins Thema einsteigen:

„Wir wissen, dass viele Frauen Gewalt erlebt haben und dass dies Auswirkungen auf die Gesundheit haben kann. Ist Ihnen schon einmal etwas gegen Ihren Willen angetan worden? Oder sind Sie schon einmal gezwungen worden, etwas zu tun, was Sie nicht wollten?" [9]

Selbst, wenn sie diese Frage nicht beantwortet, spürt die betroffene Frau, dass es möglich ist, von ihren Erfahrungen zu berichten: Statt Tabuisierung ist Offenheit möglich. Wenn sie die Frage positiv beantwortet, gibt dies eine Grundlage für weitere Unterstützung. Eine weitere mögliche Frage ist, ob die Frau Hilfs- und Unterstützungsmöglichkeiten kennt, ob es sich um ein aktuelles oder früheres Erleben handelt. Und es kann erfragt werden, ob es etwas gibt, auf das im Rahmen der Hebammenarbeit besonders geachtet werden sollte. Nicht selten können Frauen mitteilen, was für sie hilfreich ist oder was auf şie verschlimmernd wirkt.

Neben den im offenen Gespräch erfragten Informationèn können weitere Anzeichen auf Belastungen hindeuten [9]:

- fortlaufende emotionale Probleme wie Stress, Angster oder Depression

- schädigende Verhaltensweisen wie Missbrauch von Alkohol oder Drogen

- Gedanken, Pläne oder Taten von Selbstverletzung oder (versuchtem) Suizid

- Verletzungen, die sich wiederholen oder nicht überzeugend erklärt sind

- wiederholtes Auftreten sexuell übertragbarer Infektionen

- ungewollte Schwangerschaften
Einige psychische Erkrankungen werden im Zusammenhang mit Gewalt und Trauma gesehen, z.B. das Auftreten von (postpartalen) Depressionen, die Borderline Persönlichkeitsstörung oder Ess- und Angststörungen. Infolge von Traumata können selbstschädigende Verhaltensweisen beobachtet werden wie Alkohol-, Medikamenten-, Nikotin- oder Drogenkonsum. Auch Selbstverletzungen sind möglich, z.B. Schnittverletzungen [6].

Im Rahmen von Schwangerschaft und Geburt sind daneben noch andere Anzeichen zu beobachten, die sich aus den im Zusammenhang mit PTBS genannten Symptomen erklären.

\section{Mögliche Folgen}

Das Vermeiden von belastenden Situationen kann bei traumatisierten Schwangeren beispielsweise dazu führen, dass sie Vorsorgeuntersuchungen erst spät oder nicht vollständig wahrnehmen. Betroffene Frauen sind vielleicht sehr leicht aus der Ruhe zu bringen (Hyperarousal): Bereits vermeidliche Kleinigkeiten führen unter Umständen dazu, dass die Schwangere bzw. Gebärende völlig außer sich gerät und nicht mehr erreichbar ist für Zuspruch. Auf der anderen Seite kann es passieren, dass eine Frau wie erstarrt wirkt, als wäre sie gar nicht anwesend: Sie fordert nichts für sich, lässt die Geburt völlig klaglos über sich ergehen. Informationen, die ihr die Hebamme gibt, scheinen überhaupt nicht bei ihr anzukommen. Manch eine Frau verweigert eventuell notwendige Interventionen und 
Untersuchungen - eine andere wiederum lässt sie über sich ergehen: In beiden Fällen ist es für die Hebamme schwierig, die Gebärende angemessen zu betreuen. Die Frau, die in der Übererregung ist, fordert ihre ganze Aufmerksamkeit, vielleicht löst sie bei der Hebamme aber auch Abwehr aus. Früher konnte man in Geburtsberichten in solchen Fällen die Formulierung „unkooperative Gebärende“ lesen. Die zurückgezogene Frau wird hingegen häufig mit ihren Bedürfnissen übersehen. Sie scheint ja gut zurecht zu kommen. Auch dies ist nicht förderlich für eine angemessene Betreuung.

Es können auch Dissoziationen beobachtet werden: So kann es vorkommen, dass die Frau Körperempfindungen nicht wahrnehmen kann - unter Umständen auch wichtige wie Schmerzen, (vorzeitige) Wehen oder auch Schmerzen infolge von Infektionen. Gelegentlich wird sogar die Existenz der Schwangerschaft nicht wahrgenommen oder verdrängt [10].

Die körperlichen Vorgänge rund um die Geburt können leicht triggern. Dies gilt vor allem für frühere sexualisierte Gewalt, denn während der Geburt sind die gleichen Körperregionen betroffen. Das Zulassen von vaginalen Untersuchungen, der Druck, der beim Tiefertreten des Köpfchens entsteht, die Körperhaltung, die vielleicht an das Gewaltgeschehen erinnert oder das Gefühl von Hilflosigkeit und Ausgeliefertsein, all dies können Auslöser von Flashbacks sein. Auch manche Äußerung von
Hebammen und Geburtshelfern kann triggern: „Ist nicht so schlimm, ist gleich vorbei!“ - „Das kann ja gar nicht weh tun!“ Dies kann Tätersprache sein. Das Gefühl von Ohnmacht kann sich noch weiter verstärken, wenn die Gebärende sich nicht ernst genommen fühlt und ihre Wünsche nicht respektiert werden.

\begin{abstract}
Merke
Sowohl die körperlichen Vorgänge als auch die Betreuungssituation können für die Gebärende hohen - traumatischen - Stress bedeuten, der zu beobachtbaren Auswirkungen führt. Neben den beschriebenen Folgen können somatische Reaktionen festgestellt werden: starkes Schwitzen, Kreislaufprobleme, Übelkeit, Zittern, blasse Gesichtsfarbe, Schwindel und anderes. Diese Symptome können ihre Ursache auch im Gebärprozess selbst haben. Für die Hebamme ist es allerdings wichtig, ein Trauma als mögliche Ursache in Betracht zu ziehen, im Sinne der Differenzialdiagnose.
\end{abstract}

\section{Unterstützung durch Hebammen}

Jede Frau sollte so unterstützt werden, als hätte sie traumatische Erlebnisse gemacht. Denn es kann niemals sicher gesagt werden, welche Erfahrungen jemand in der Vergangenheit überlebt hat, auch nicht durch gezieltes Nachfragen. Andererseits profitiert jede Gebärende von einer traumasensiblen Arbeitsweise.

Traumasensibel meint in diesem Sinne nicht eine spezielle Methode oder Technik. Zunächst einmal ist darunter ein Verständnis für die Folgen von verletzenden Erfahrungen und die Fähigkeit zu verstehen, diese Auswirkungen auf den Kontext von Schwangerschaft, Geburt und Mutterschaft zu übertragen. Ziel der Hebamme sollte es sein, der Gebärenden zu fördernden Gegenerfahrungen zu verhelfen - Unterstützung statt Gewalt, Selbstwirksamkeit statt Ohnmacht und Hilflosigkeit, Selbstbestimmung, Stabilität und Autonomie statt Kontrollverlust.

Konkret betrifft eine traumasensible Arbeitsweise von Hebammen insbesondere folgende Bereiche:

\section{Kommunikation}

Die Wortwahl ist sorgsam zu treffen. Neben der Vermeidung von potenziell triggernden Aussagen (,machen Sie die Beine breit“) ist es hilfreich, wenn Frauen in der Wahrnehmung ihrer eigenen Empfindungen bestärkt werden. Die Formulierung, „das tut jetzt nicht weh, oder?“, suggeriert, dass es nicht weh tun kann. Dies kann zu einer weiteren Verunsicherung führen - die Frau denkt womöglich, in diesem Fall stimme mit ihr etwas nicht, falls sie es doch schmerzhaft empfindet. Besser sind offene Fragen: „Wie fühlt sich das für Sie an?“ - das lässt der Frau Raum für eigene Wahrnehmungen und bietet die Möglichkeit, diese zu äußern. 
Der nonverbalen Kommunikation kommt ebenfalls große Bedeutung zu. Hebammen sind gehalten, auf Augenhöhe zu kommunizieren. Dies bedeutet einerseits, dass nicht von oben herab mit den Frauen gesprochen werden soll. Dies würde ein Machtgefälle signalisieren und kann an früheres Gewalterleben erinnern. Sich auf Augenhöhe zu begeben, bedeutet z.B., sich auf einen Stuhl neben das Kreißbett zu setzen oder sich mit auf den Boden zu knien. Augenhöhe bedeutet noch etwas: Hier treffen zwei Expertinnen aufeinander - die Hebamme als die Expertin mit dem Fachwissen im Gebärzimmer und die Frau in den Wehen als die Expertin für ihren Körper und ihr Leben. Erst die Kombination der Expertisen ergibt ein rundes Bild. Dies sollten Hebammen stets beachten. Sätze wie, „Das kann doch gar nicht sein, dass Sie schon Pressdrang haben ... dass die Wehen schon kräftig sind ...", sprechen der Frau in den Wehen ihr eigenes Expertinnenwissen ab und entmündigen sie.

\section{Stabilität und Sicherheit}

Gebären kann für eine traumatisierte Frau hohe Unsicherheit und großen Stress bedeuten. Sie begibt sich in eine unbekannte Situation und soll Menschen vertrauen, die sie kaum kennt. Sie hat keine Kontrolle über die Körperarbeit, die Wehen passieren einfach. Die Aufgabe der Hebamme ist es, der Frau größtmögliche Sicherheit zu vermitteln, sodass sie sich auf die Geburtsarbeit konzentrieren kann. Leitgedanke dabei: Was benötigt sie für ihre äußere und innere Sicherheit?

Wenn möglich kann die Hebamme erfragen, was dafür wichtig ist, z.B. dass Türen geschlossen bleiben und nur auf Aufforderung der Raum betreten werden kann, dass die Frau weiß, wer bei der Geburt anwesend ist und dass ihre Wünsche berücksichtigt werden. Wenn die Hebamme die Schwangere schon vor der Geburt kennenlernt, bietet es sich an, schon im Vorfeld der Geburt mit ihr darüber zu sprechen, was sie ängstigt beziehungsweise was ihr hilft. Dies kann dann in einem Geburtsplan zusammengefasst werden [10].

Sicherheit und Stabilität kann die Hebamme auch durch ihre Stimme vermitteln. Gerade wenn sie bemerkt, dass eine Frau in Stress gerät, Atmung und Herzschlag schneller werden oder die Gebärende droht, in eine Dissoziation wegzudriften, dann ist es hilfreich, mit klarer, deutlicher und angemessen lauter Stimme zu sprechen. In keinem Fall ist damit ein Schreien gemeint, sondern eher eine klare Ansprache: „Frau Müller, schauen Sie mich an: ich möchte, dass Sie sich jetzt aufsetzen!“ In solchen Momenten ist anders als sonst - Klarheit und Führung gefragt.

Dissoziationen und Flashbacks während der Geburt sind nicht selten. Betroffene verlieren den Kontakt zum jetzigen Moment, sie rutschen in ein früheres Erleben. Für Hebammen, die wenig Erfahrung und Wissen zum Thema Trauma haben, kann dies beängstigend sein. Wenn klare Ansprache allein nicht reicht, um die Frau ins Hier und Jetzt zurückzuholen, kann es helfen, sie in Aktion zu bringen: Die Hebamme bittet die Frau z.B., die Hände zu krallen oder zu strecken, sich hinzustellen, in ihrer Begleitung durch den Raum zu gehen und mit den Füssen auf den Boden zu stampfen. Eine andere Möglichkeit besteht darin, die Gegenwart mit allen Sinnen wahrzunehmen: Die Hebamme lässt die Frau beschreiben, was sie sieht, hört, riecht oder schmeckt. Dazu kann sie ihr ein Pfefferminzbonbon anbieten - der scharfe Geschmack hilft, sich wieder in der Gegenwart zu orientieren. Auch der Duft einer Aromalampe ist nutzbar: Kann sie ihn riechen? Wie findet sie den Geruch?

Vorsichtig ist mit Berührungen umzugehen. Es kann sehr unangenehm sein, von Fremden berührt zu werden. Für Traumatisierte kann eine solche Berührung zum Auslöser eines Flashbacks werden. Gerade, wenn die Hebamme das Gefühl hat, die betreute Frau dissoziiert oder zeigt gerade deutliche Stresssymptome, kann eine ungefragte Berührung die Situation eher verschlimmern. Stattdessen kann die Hebamme die Frau zur Aktion auffordern: „Frau Meier, greifen Sie meine Hand und drücken Sie fest zu!“

\section{Vaginale Untersuchungen}

Wenn eine Untersuchung notwendig ist, sollte diese vorbereitet werden: Die Hebamme erklärt der Frau, weshalb die Untersuchung notwendig ist. Sie hilft der werdenden Mutter in eine Position, die ihr angenehm ist. Sie kündigt an, was als nächstes passiert („Sie werden jetzt meine Finger an Ihrer Vulva spüren und dann werden Sie merken, wie ich den Finger in die Vagina einführe“). Sie bleibt mit der Frau in Kontakt, sowohl verbal als auch mit Blickkontakt. Nur so kann die Hebamme Stresszeichen wie Blässe oder übermäßiges Schwitzen wahrnehmen und entsprechend darauf reagieren. Sie überlässt der Frau die Führung! Diese gibt das Tempo vor und muss wissen, dass sie die Untersuchung jederzeit abbrechen kann. Im Anschluss an die Untersuchung hilft die Hebamme ihr wieder in eine entspannte Position und beim Ankleiden. Hilfreich ist es, zu fragen, ob bei der nächsten Untersuchung etwas geändert werden sollte.

\section{Operative Geburtsbeendigung}

Eine operative Geburtsbeendigung stellt für jede Frau eine große Herausforderung dar. In dieser hochunsicheren Situation wird die Frau einen Anker brauchen, der sie im Hier und Jetzt hält. Dafür bleibt die Hebamme in jedem Fall im Kontakt mit der Gebärenden. Soweit möglich sollte die Gebärende in Entscheidungen mit einbezogen werden. In Notfällen fehlt oft die Zeit, angemessen zu erklären und die Frauen (und die Partner) mitzunehmen. Zumindest im Nachhinein sollte dann die Möglichkeit einer Nachbesprechung gegeben werden. Die Hebamme spricht der Mutter ihren Respekt aus für die Leistung, die sie erbracht hat, vor allem unter diesen schwierigen Umständen. 
Eine primäre Sectio scheint manchmal ein einfacher Weg zu sein, die Unwägbarkeiten einer vaginalen Geburt zu vermeiden. Dies kann, muss aber keinesfalls eine Lösung darstellen. Sowohl die Sectio als auch die vaginale Geburt stellen hohe Anforderungen an Hebammen und Gebärende. Es sollte im Einzelfall individuell abgewogen werden, welche Ängste bestehen und wie diesen begegnet werden kann.

\section{Unterstützung für Hebammen}

Die Begleitung von traumatisierten Menschen ist eine wichtige, aber anstrengende Tätigkeit, die hohe Anforderungen an Hebammen stellt. Diese Arbeit kann sehr bereichernd sein, aber sie birgt auch Risiken. Es gibt eine „natürliche, vorhersehbare, behandelbar und verhinderbare unerwünschte Folge der Arbeit mit leidenden Menschen“ [9]. So kann es passieren, dass Symptome auftauchen, die denen einer PTBS entsprechen [14]. Um gesund zu bleiben, ist es notwendig, dass Hebammen lernen, für sich selbst genauso Sorge zu tragen wie für ihre Patientinnen. Dafür brauchen sie entsprechende Arbeits- und Rahmenbedingungen. Die Möglichkeit zu Fortbildung, kollegialer Beratung und Supervision sind unabdingbar, genauso wie ein Dienstplan, der Pausen, einen pünktlichen Feierabend und eine vernünftige Freizeitgestaltung zulässt.

Hebammen sind achtsam ihren Patientinnen gegenüber, die gleiche achtsame Wahrnehmung sollten sie für sich pflegen. Es kann passieren, dass sie selbst merken, wie sie den Kontakt zum Hier und Jetzt verlieren. Dann wird es auch ihnen helfen, sich mit allen Sinnen zu reorientieren, den Boden unter den Füßen zu spüren, Geräusche wahrzunehmen und sich visuelle Reize bewusst zu machen.

Hebammen müssen lernen, sich abzugrenzen, um langfristig für ihre Patientinnen eine empathische Unterstützung bieten zu können. Ihre Aufgabe ist die fachliche Unterstützung als Hebamme, die sensibel für Auswirkungen und Dynamiken von Traumata ist. Nicht mehr und nicht weniger. Für jegliche weitere Unterstützung gibt es Fachberatungsstellen, an die Frauen weitervermittelt werden können.

\section{Über die Autorin}

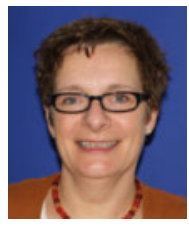

Martina Kruse, B.A., M.A., ist Hebamme und Familienhebamme, Systemische Beraterin (SG) und Traumazentrierte Fachberaterin (DeGPT). Sie schrieb ihre Masterthesis zum Thema „Traumasensible Haltung in der Hebammenarbeit - Entwicklung einer Fortbildungsreihe". Sie bietet Fortbildungen an mit Grundlagenwissen zum Thema Trauma/Psychotraumatologie sowie zu den Auswirkungen von traumatischem Stress allgemein und im Kontext von Schwangerschaft und Geburt. In Kürze erscheint ihr Fachbuch zum Thema im Hippokrates Verlag.
Korrespondenzadresse

\section{Martina Kruse}

Kyllburgerstr. 7

50937 Köln

martina.kruse@gmx.net

Literatur

[1] Beck CT. Birth Trauma. In the eye of the Beholder. In: Nursing Research 2004; 1: 28-35, DOI: 10.1097/00006199200401000-00005: 28

[2] European Union Agency for Fundamental Rights. Gewalt gegen Frauen:eine EU-weite Erhebung. Wien; 2014

[3] Figley CF. Mitgefühlserschöpfung. Der Preis des Helfens. In: Hudnall Stamm B, Hrsg. Sekundäre Traumastörungen. Wie Kliniker, Forscher \& Erzieher sich vor traumatischen Auswirkungen ihrer Arbeit schützen können. Paderborn: Junfermann; 2002: 41-59

[4] Fischer G, Riedesser P. Lehrbuch der Psychotraumatologie. 4. Aufl. München: Reinhardt; 2009: 84

[5] Flatten G, Gast U, Hofmann A, et al. S3 - Leitlinie Posttraumatische Belastungsstörung. Trauma \& Gewalt 2011; 3: $202-210$

[6] Hantke L, Görges H-J. Handbuch Traumakompetenz. Basiswissen für Therapie, Beratung und Pädagogik. Paderborn: Junfermann; 2012

[7] Leeners B, Richter-Appelt H, Schönfeld K, et al. Schwangerschaft und Mutterschaft nach sexuellen Missbrauchserfahrungen im Kindesalter: Auswirkungen und Ansätze zu einer verbesserten Betreuung bei Schwangerschaft, Geburt, Still- und früher Neugeborenenzeit. In: Deutsches Ärzteblatt 2003; 11: A715-A719

[8] Reddemann L, Dehner-Rau C. Trauma heilen. Ein Übungsbuch für Körper und Seele. 4., vollst. überarb. Aufl. Stuttgart: Trias; 2013

[9] Robert Koch-Institut. Gesundheitliche Folgen von Gewalt unter besonderer Berücksichtigung von häuslicher Gewalt gegen Frauen. Gesundheitsberichtserstattung des Bundes. Berlin: Robert Koch-Institut; 2008.

[10] S.I.G.N.A.L. e. V. Gesundheitliche Versorgung von Frauen, die Gewalt in der Paarbeziehung oder sexuelle Gewalt erfahren. Klinisches Handbuch der WHO. Feldtest-Version. Berlin; 2014

[11] Simkin P, Klaus P. Wenn missbrauchte Frauen Mutter werden. Die Folgen früher sexueller Gewalt und therapeutische Hilfen. Stuttgart: Klett-Cotta; 2015

[12] United Nations. Distr. GENERAL. A/RES/48/104. 23 February 1994. Online verfügbar: http://www.un.org/en/ga/search/ view_doc.asp?symbol=A/RES/48/104\&Lang=E\&Area=RESOL UTION

[13] Wahlberg A, Andreen Sachs M, Johannesson K et al. Post-traumatic stress symptoms in Swedich obstetricians and midwives after severe obstetric events: a cross-sectional retrospective survey. BJOG 2016, DOI: 10.1111/14710528.14259

[14] Wessel J. Die verdrängte Schwangerschaft. Akademos: Hamburg; 2007 
